\title{
Experiencias en la Aplicación de un Ciclo de Mejora en las prácticas de Circuitos Electrónicos Digitales
}

\section{Experiences in the Application of an Improvement Cycle in the practices of Digital Electronic Circuits}

Betsaida AleXANDRe Barajas

ORCID: https://orcid.org/0000-0002-5200-7103

Universidad de Sevilla

Departamento de Tecnología Electrónica

balexandre@us.es

DOI: http://dx.doi.org/10.12795/9788447231003.026

Pp.: 555-572 


\section{Contexto educativo}

La experiencia de diseño y aplicación de este Ciclo de Mejora en el Aula (CIMA) se desarrolló en las Practicas de Laboratorio de la asignatura Circuitos Electrónicos Digitales (CED). Es una asignatura de primer curso que se imparte en el primer cuatrimestre de forma simultánea en varias titulaciones del Grado en Ingeniería Informática: Ingeniería de Computadores, Ingeniería de Tecnologías e Ingeniería del Software.

En las Prácticas de Laboratorio los alumnos deben montar circuitos previamente explicados en clase, y usando los equipos de laboratorio toman las medidas que les permitan comprobar el funcionamiento de los circuitos. Las sesiones de practica tienen una duración de 2 horas. Cada una de estas, parte de unos enunciados o boletines que el profesor ha proporcionado con antelación. Previo a la práctica, el alumno debe haber leído los enunciados, así como hacer un estudio teórico y el esquemático de los circuitos para llegar al laboratorio a montarlos para poder tomar las medidas. Pero la realidad es que un gran porcentaje de alumnos carece de la motivación para revisar conscientemente los enunciados antes de llegar al laboratorio, por lo que al inicio de las sesiones para ponerlos en contexto el profesor a través de una pequeña presentación explica lo que se va a hacer en la práctica y les indica los esquemas que deben montar. Los alumnos parten de estos esquemáticos y montan los circuitos. En la gran mayoría de los casos ocurre que el circuito no funciona adecuadamente y como los estudiantes no han asimilado el esquemático y menos aún el funcionamiento correcto del circuito, dedican la mayor parte del tiempo a buscar donde está el fallo y a solucionarlo. Generalmente no pueden tomar medidas, ni culminar con éxito las practicas. Esto genera estrés en el alumnado y en el profesorado, ya que no se cumplen los objetivos.

Ciclos de Mejora en el Aula (2020). Experiencias de Innovación Docente de la US Esta obra se distribuye con la licencia Creative Commons 


\section{Diseño previo del Ciclo de Mejora en el Aula}

Con la intención de mejorar la situación antes mencionada, se plantea el diseño de un Ciclo de Mejora en el Aula (CIMA) en las Practicas de Laboratorio de CED. Primero se definieron los contenidos que se deseaba que los estudiantes aprendieran durante el CIMA a través de problemas claves que describen los aprendizajes deseables; luego se diseñó el Modelo Metodológico posible a emplear, posteriormente se proyectó la secuencia de las actividades a realizar y por último se diseñaron los cuestionarios inicial-final a través de los cuales se podía hacer un seguimiento de la evolución del aprendizaje de los alumnos. A continuación, se detallan cada una de estas fases.

\section{Mapa de Contenidos}

Generalmente cuando se programan los contenidos se hace referencia a conceptos de la disciplina, pero la realidad es que no es lo único que se debe tener en cuenta. Por lo que se diferenciaron tres grupos de contenidos: los conceptuales, estos se refieren a conceptos y principios de la disciplina; los procedimentales, aqui se engloban las habilidades y destrezas, bien sean psicomotrices intelectuales y finalmente los actitudinales, los cuales están relacionados con valores y actitudes (Porlán, 2017). También se propuso una organización en red, tramas o mapas (García, 1998), en donde también se encuentran contenidos cuya función es la de organizar a otros de menor grado, los cuales hemos identificado como contenidos estructurantes (Porlán, 2017).

Los contenidos se refieren siempre a preguntas o problemas que nos hacemos para comprender y actuar en la realidad (Porlán, 2017). Para el CIMA se plantearon 4 problemas claves. En la Figura 1, se muestra el mapa de contenidos y problemas asociado a las sesiones del CIMA.

Ciclos de Mejora en el Aula (2020). Experiencias de Innovación Docente de la US Esta obra se distribuye con la licencia Creative Commons 


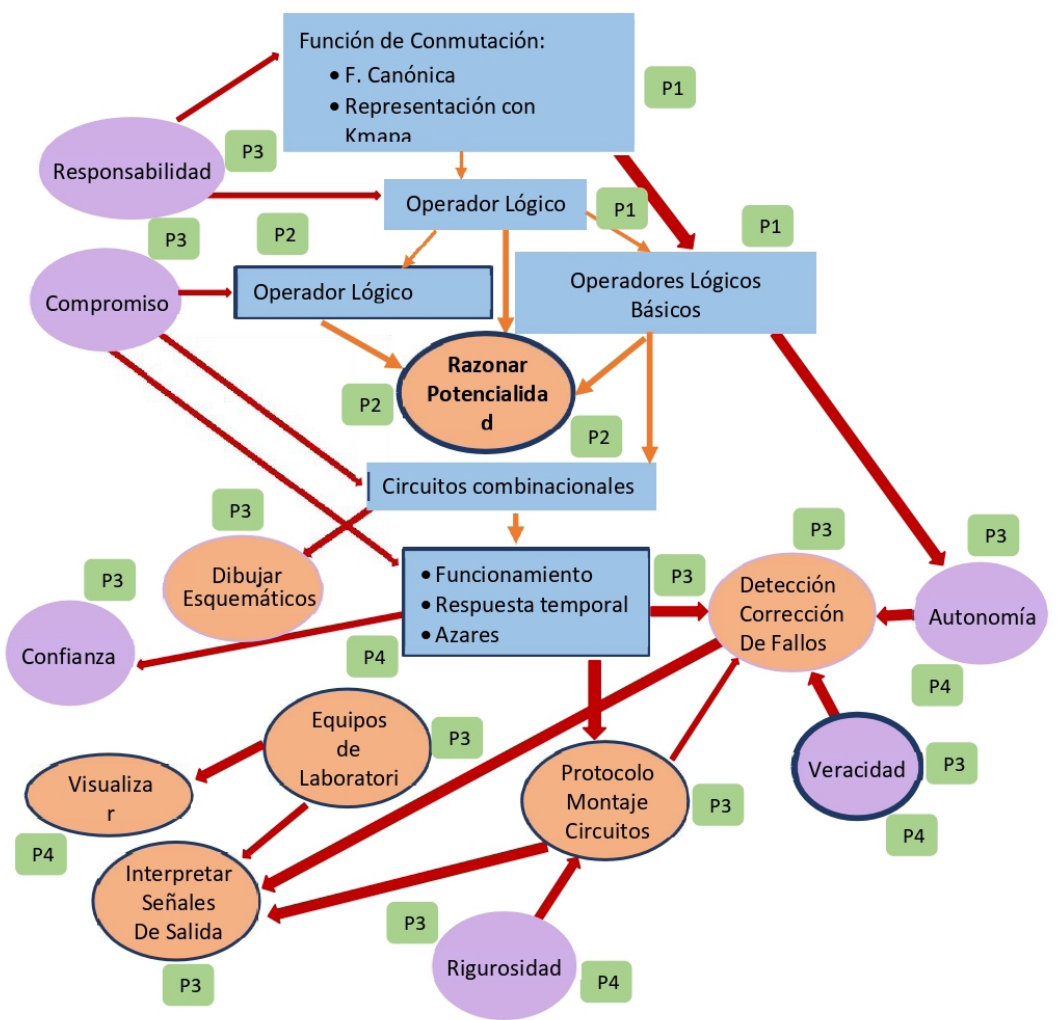

Contenidos Conceptuales

Contenidos

Actitudinales

Contenidos

Procedimentales

Contenidos Estructurantes

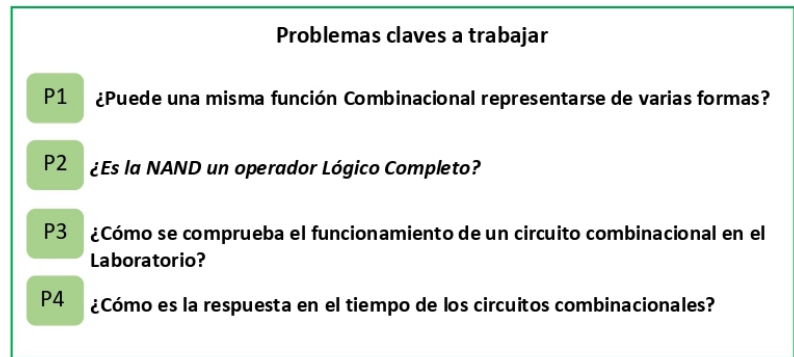

Figura 1. Mapa de contenidos asociados a las sesiones del CIMA

\section{Modelo metodológico empleado}

El modelo metodológico comprende las pautas, rutinas y esquemas que utilizamos en el aula. El modelo basado en la transmisión es el predominante en la docencia universitaria, pero para este CIMA se planteó un cambio

Ciclos de Mejora en el Aula (2020). Experiencias de Innovación Docente de la US (c) $\odot$ Esta obra se distribuye con la licencia Creative Commons 
basado en comenzar con los estudiantes en lugar de con la disciplina, de manera que permita a los alumnos construir su propio aprendizaje, orientándolos a desarrollar sus propias explicaciones y a defenderlas, dirigiéndolos para conseguir el razonamiento disciplinar (Bain, 2007). En la Figura 2 se muestra el modelo metodológico diseñado para el CIMA. Previo a cada sesión, el estudiante desarrolla un cuestionario inicial (Cl) el cual es enviado al profesor antes de comenzar la práctica. Cada sesión se inicia creando un contexto intrigante que plantea un problema (C/P), seguidamente los alumnos realizan un foro de debate donde plantean sus ideas (IAG), posteriormente se plantea la primera actividad de contraste (AC1) en donde se ponen en común las ideas y son orientadas por el profesor, luego los alumnos de forma individual organizan sus ideas (IA) para después desarrollar la práctica de Laboratorio (AC2), la cual constituye la segunda actividad de contraste. Finalmente se plantea realizar dentro de cada sesión, un cuestionario final $(\mathrm{Cl}-\mathrm{F})$ y las conclusiones por parte del profesor.

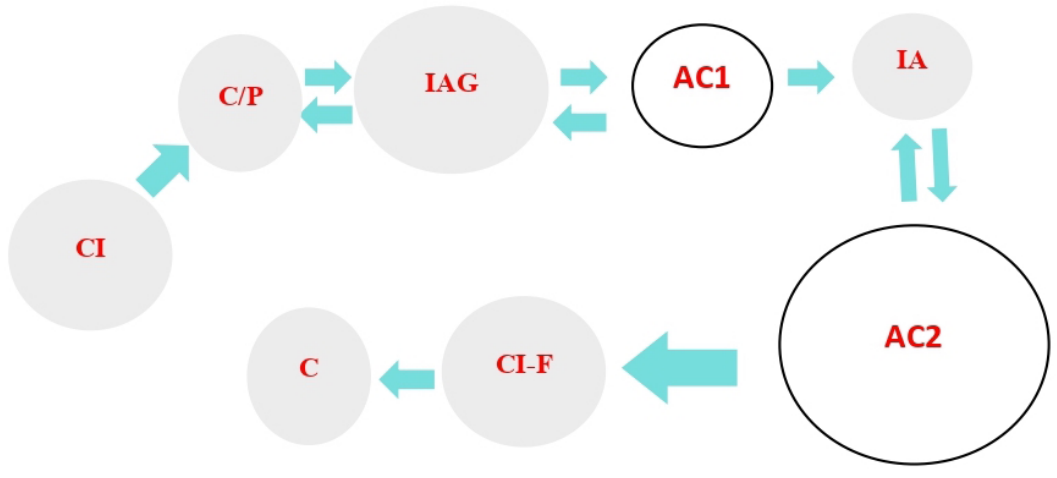

Figura 2. Modelo Metodológico empleado en el CIMA

Secuencia de actividades del CIMA

Para el CIMA se diseñó una secuencia de actividades para cada una de las sesiones, prácticamente iguales, cuya única diferencia estaba en el contexto o problema clave a trabajar. La Tabla 1 muestra la secuencia de actividades para cada sesión.

Ciclos de Mejora en el Aula (2020). Experiencias de Innovación Docente de la US Esta obra se distribuye con la licencia Creative Commons 
Tabla 1. Secuencia de actividades para cada sesión del CIMA

\begin{tabular}{|c|c|c|c|}
\hline 1 & Contexto y Problema & Fase: $\mathrm{C} / \mathrm{P}$ & $\mathrm{T}=5 \mathrm{~min}$ \\
\hline \multicolumn{4}{|c|}{$\begin{array}{l}\text { Explicación del Contexto: Somos un Laboratorio muy prestigioso en el } \\
\text { campo de Testado de Chips. El docente es el coordinador del Laboratorio y } \\
\text { los alumnos los Ingenieros de Testado. Han contratado al Laboratorio para } \\
\text { diseñar un sistema de testado. } \\
\text { Explicación del problema clave, y el conjunto de preguntas que se necesita } \\
\text { resolver }\end{array}$} \\
\hline \multicolumn{4}{|c|}{ Recursos: Presentación en PDF y video Beem } \\
\hline 2 & Grupos de Trabajo & Fase: IAG & $\mathrm{T}=30 \mathrm{~min}$ \\
\hline \multicolumn{4}{|c|}{$\begin{array}{l}\text { Se forman grupos de trabajo } \\
\text { Discuten en el grupo las respuestas que los alumnos han hecho en el } \\
\text { cuestionario inicial. Y formulan una o dos hipótesis para resolver el } \\
\text { problema } \\
\text { Establecen respuestas en común. }\end{array}$} \\
\hline \multicolumn{4}{|c|}{ Recursos: Foro de Debate } \\
\hline 3 & Actividad de Contraste 1 & Fase: AC1 & $\mathrm{T}=15 \mathrm{~min}$ \\
\hline \multicolumn{4}{|c|}{$\begin{array}{l}\text { Un representante de cada grupo interviene y expone las respuestas a } \\
\text { las preguntas, defendiendo el por qué su grupo ha respondido de esa } \\
\text { manera. } \\
\text { Al final de cada intervención, animo al grupo para que de sus } \\
\text { observaciones. } \\
\text { El docente da sus observaciones orientando hacia la respuesta adecuada. }\end{array}$} \\
\hline \multicolumn{4}{|c|}{ Recursos: Exposición y Debate } \\
\hline 4 & Ideas de los Alumnos & Fase: IA & $T=5 \mathrm{~min}$ \\
\hline \multicolumn{4}{|c|}{$\begin{array}{l}\text { Los alumnos ponen en limpio sus ideas de cara a lo que se va a hacer en } \\
\text { la practica }\end{array}$} \\
\hline \multicolumn{4}{|c|}{ Recursos: Apuntes } \\
\hline 5 & Desarrollo de la Practica de Laboratorio & Fase: AC2 & $\mathrm{T}=50 \mathrm{~min}$ \\
\hline \multicolumn{4}{|c|}{$\begin{array}{l}\text { Montan los circuitos y les hacen las mediciones para probar las hipótesis } \\
\text { planteadas }\end{array}$} \\
\hline
\end{tabular}

Ciclos de Mejora en el Aula (2020). Experiencias de Innovación Docente de la US Esta obra se distribuye con la licencia Creative Commons Reconocimiento-NoComercial-SinObraDerivada $\quad 4.0$ Internacional (CC BY-NC-ND 4.0.) 


\begin{tabular}{|l|l|l|l|}
\hline $\mathbf{6}$ & Cuestionario Inicial-Final & Fase: $\mathbf{C F}$ & T=10min \\
\hline \multicolumn{2}{|l|}{ Aplicación del Cuestionario Final } & \multicolumn{2}{l|}{} \\
\hline \multicolumn{2}{|l|}{ Recursos: Cuestionario } & Fase: $\mathbf{C}$ & T=5min \\
\hline $\mathbf{7}$ & Conclusiones & & \\
\hline \multicolumn{4}{|l|}{ Conclusiones del docente sobre la actividad } \\
\hline \multicolumn{2}{|l|}{ Recursos: Exposición } \\
\hline
\end{tabular}

Cuestionarios inicial y final

Para centrar la atención de los estudiantes en aspectos específicos del problema es necesario plantear una serie de preguntas con las que se mantenga la curioszdad y se pueda construir el conocimiento (Bain, 2007). De igual forma, para conocer y analizar los modelos mentales de los estudiantes a través de los cuales es posible inferir la evolución del aprendizaje es necesario utilizar instrumentos como cuestionarios con los que se obtiene información que permite inferir la progresión del conocimiento (Porlán, 2017). En base a esto se diseñaron 4 cuestionarios iniciales para ser aplicados previamente en cada sesión y al final. A modo de ejemplo las preguntas que se hicieron en el cuestionario inicial de la segunda sesión son las siguientes:

1. ¿Cómo implementarías el circuito combinacional de la siguiente función usando los Operadores Lógicos Básicos? Ten en cuenta que las variables están en único raíl. Puedes dibujar el circuito a mano y poner la foto.

$$
\mathbf{f}=a b+c \bar{a}
$$

Ciclos de Mejora en el Aula (2020). Experiencias de Innovación Docente de la US Esta obra se distribuye con la licencia Creative Commons Reconocimiento-NoComercial-SinObraDerivada Internacional (CC BY-NC-ND 4.0.) 
2. ¿Cómo implementarías el circuito combinacional de esta función usando exclusivamente puertas NAND de 2 entradas? Ten en cuenta que las variables están en único raíl. Puedes dibujar el circuito a mano y poner la foto.

3. En relación con las dos respuestas anteriores: Indica y explica todas las diferencias que encuentras

4. Imagina que estas en el Laboratorio, para el circuito de solo compuertas NAND: ¿Qué harías para ver simultáneamente la señal a y la salida f? Intenta convencerme con tu argumentación

\section{Diario de las sesiones}

A pesar de las condiciones especiales de docencia online que vivimos actualmente por la pandemia, mi asignatura no estaba enmarcaba en esta forma, debido a que en los grados de Ingeniería Informática las prácticas de laboratorio siguen siendo presenciales, por lo que todo mi CIMA estaba diseñado para hacerlo de esta forma presencial. Pero los días previos a la aplicación me indicaron que tenía que estar en confinamiento preventivo, así que sobre la marcha diseñe la forma de hacerlo online. Cuando le comunique a mi coordinador que estaba confinada, me dijo que lo mejor era que la práctica se cancelara porque debía ser presencial, casi me da algo, después de haber trabajado bastante en la preparación de las actividades, me hacía ilusión esta nueva forma de trabajar y tenía grandes expectativas; así que me llené de valor y le escribí que yo quería hacer las sesiones de forma online porque tenía preparado los contenidos de la práctica para la aplicación de un ciclo de mejora en la docencia; gracias a esto él me aprobó que trabajara de esta forma.

A la primera sesión asistieron 12 de los 15 alumnos. Previamente, el cuestionario inicial lo había aplicado de forma online. Estaba super nerviosa ya que no había trabajado Ciclos de Mejora en el Aula (2020). Experiencias de Innovación Docente de la US
Esta obra se distribuye con la licencia Creative Commons 
de forma online desde junio y más porque la parte practica la tenía que hacer a través de simulaciones. Presenté el contexto en el que íbamos a trabajar junto con la metodología que íbamos a usar. Les recordé que la aplicación de este proceso requería de un compromiso por su parte y de una participación. Seguidamente forme los grupos (de tres personas), a través de la plataforma online y les indique que durante los siguientes 30 minutos ellos iban a discutir las respuestas del cuestionario e iban a tratar de llegar a acuerdos para después ponerlos en común, también les recordé que alguno de los integrantes del grupo tenía la función de secretario. Yo me pasé por cada grupo y fui resolviendo dudas sobre algunas preguntas que no tenían claras. Me llamo la atención la autonomía y el interés con el que estaban trabajando. A mi parecer hubo grupos que terminaron muy pronto ya que tenían muy recientes sus respuestas en el cuestionario online. Debo reconocer que todo esto lo hice gracias a la sesión que habíamos tenido el día anterior sobre Enseñanza Virtual en el curso de Docencia Universitaria. La puesta en común era mi primera actividad de contraste, pedía a un grupo que contestara a una pregunta y los otros comentaban después si habían encontrado más información o la habían enfocado de otra forma. En esta actividad yo había previsto 15 minutos, pero tardamos el doble. Por muchos factores, ellos tenían que compartir sus pantallas para mostrar los dibujos y esto consume más tiempo. Además, si me descuidaba me ponía a hablar y a explicar, mi tendencia a las clases magistrales se reflejaba, y la de los alumnos a dejarme hacerlo; lo difícil de cambiar hábitos que he practicado durante muchos años. Me di cuenta de lo complicado que es dirigir una puesta en común. Me percate que había planificado poco tiempo debido a mi inexperiencia en este tipo de actividades; ya que los alumnos tienen un ritmo diferente en las exposiciones por lo que necesitan más tiempo. El darme cuenta de que había consumido el doble de tiempo me causo cierto nerviosismo, pero me calme y continúe con la

Ciclos de Mejora en el Aula (2020). Experiencias de Innovación Docente de la US Esta obra se distribuye con la licencia Creative Commons 
planificación. La siguiente actividad que también me puso en tensión inicialmente, fue el desarrollo de la práctica, ya que tenía que explicarles como funcionada el simulador, pero en realidad los estudiantes lo hicieron con mucho interés. De nuevo resulto muy corto el tiempo que estime para esta actividad. Este ha sido uno de mis mayores problemas en la aplicación de este CIMA.

La segunda sesión también fue de forma online. El cuestionario inicial para esta sesión también se lo había enviado previamente En esta ocasión asistieron 9 alumnos. En general, durante esta sesión las actividades funcionaron, me sentí a gusto en general. Como en todas las sesiones del CIMA, comencé la clase creando un entorno de aprendizaje crítico y natural; con la intensión de que los alumnos despertarán su curiosidad, específicamente a través de una pregunta intrigante (Bain,2007). Alternamos trabajo en equipo, puesta en común, practica online y de vez en cuando un resumen por parte de la profesora. Al igual que la sesión anterior me quedé corta de tiempo, pero ya no me puse nerviosa.

La tercera sesión fue presencial ya había salido del aislamiento y asistieron 9 alumnos. Previamente les había enviado el cuestionario. Seguimos trabajando del mismo modo, realizando grupos de debate, que ya ellos llaman foros de debate, puesta en común y práctica. Si es necesario la profesora muestra un nuevo detalle que no haya aparecido. En esta ocasión para la puesta en común dividí el pizarrón en 3 partes y cada secretario dibujo allí los circuitos, todo el grupo apoyaba a cada secretario esta parte me gustó mucho, los alumnos participaron de forma muy interesada a tal punto que al final de la clase se quedaron quince minutos trabajando.

La cuarta y última sesión también fue presencial, en esta ocasión asistieron 12 alumnos. Antes de la sesión les había enviado el cuestionario inicial, la aplicación de este instrumento me gustó mucho, ya que sentí a los alumnos muy libres de dar sus respuestas, aunque en las practicas 
normalmente se le exigía al alumno un estudio teórico previo, pude observar que los cuestionarios iniciales motivaban más a los estudiantes. El desarrollo de la sesión siguió la misma tónica que en las anteriores; estudiantes motivados y trabajando. Pero al desarrollar la parte práctica algunos alumnos tuvieron bastantes problemas con el funcionamiento del circuito y más aún en la detección y solución de fallos, de verdad esto me dejo un mal sabor, pero la realidad es que podría decirse que los alumnos tenían que superar varios obstáculos. En líneas generales me siento muy satisfecha por la participación y la actitud de los alumnos.

\section{Evaluación del aprendizaje de los alumnos}

Para poder valorar el aprendizaje del estudiante durante el CIMA, en mi diseño tenía previsto aplicar de nuevo al final de cada sesión el cuestionario inicial, pero debido a la falta de tiempo que siempre tenía en todas las sesiones, mientras estaba aplicando el CIMA, tome la decisión de asignar una tarea calificada con 4 preguntas que englobaba todo el cuestionario inicial. El indicarles a los estudiantes que la tarea la iba a evaluar los motivo para hacerla más detallada.

De esta manera pude evaluar los cambios en los modelos mentales de los estudiantes en relación con el aprendizaje adquirido de los contenidos conceptuales, procedimentales y actitudinales. Solo 9 alumnos coincidieron en responder todos los cuestionarios iniciales y la tarea final. El analizar esto me permitió reconocer diferentes modelos de respuestas para cada pregunta, cada modelo se relacionó con una letra $(A, B, C, D, E)$ donde $A$ es el modelo mental que se acerca más a la respuesta ideal de las preguntas, y de D o E se relaciona al modelo mental más alejado. La Tabla 4 muestra los modelos para las 4 preguntas globales, para las preguntas 3 y 4 solo aparecieron 4 modelos, mientras que para la primera y la segunda pregunta se agruparon en 5 modelos.

Ciclos de Mejora en el Aula (2020). Experiencias de Innovación Docente de la US Esta obra se distribuye con la licencia Creative Commons 
Tabla 4. Definición de los modelos de respuestas para las 4 preguntas globales

\begin{tabular}{|c|c|c|c|c|}
\hline Modelo & Pregunta 1 & Pregunta 2 & Pregunta 3 & Pregunta 4 \\
\hline A & $\begin{array}{l}\text { Relaciona la } \\
\text { función con las } \\
\text { formas Canónica y } \\
\text { Normalizadas }\end{array}$ & $\begin{array}{l}\text { Establece } \\
\text { diferencias } \\
\text { entre la NAND y } \\
\text { los Operadores } \\
\text { Lógicos }\end{array}$ & $\begin{array}{l}\text { Identifica y } \\
\text { soluciona de } \\
\text { forma autónoma } \\
\text { los fallos que } \\
\text { ocurren durante } \\
\text { las mediciones }\end{array}$ & $\begin{array}{l}\text { Reconoce el } \\
\text { azar estático y } \\
\text { las condiciones } \\
\text { de la señal de } \\
\text { entrada para } \\
\text { visualizarlo }\end{array}$ \\
\hline B & $\begin{array}{l}\text { Identifica } \\
\text { las formas } \\
\text { Normalizadas } \\
\text { de las funciones } \\
\text { combinacionales }\end{array}$ & $\begin{array}{l}\text { Reconoce la } \\
\text { NAND como un } \\
\text { operador lógico } \\
\text { completo }\end{array}$ & $\begin{array}{l}\text { Comprende } \\
\text { los criterios de } \\
\text { rigurosidad en } \\
\text { el montaje de } \\
\text { circuitos }\end{array}$ & $\begin{array}{l}\text { Identifica los } \\
\text { equipos del } \\
\text { laboratorio } \\
\text { donde se miden } \\
\text { señales. }\end{array}$ \\
\hline C & $\begin{array}{l}\text { Identifica las } \\
\text { formas Canónicas } \\
\text { de las funciones } \\
\text { combinacionales }\end{array}$ & $\begin{array}{l}\text { Dibuja el circuito } \\
\text { combinacional } \\
\text { utilizando solo } \\
\text { el operador } \\
\text { lógico NAND }\end{array}$ & $\begin{array}{l}\text { Reconoce el } \\
\text { funcionamiento } \\
\text { de los Equipos del } \\
\text { Laboratorio }\end{array}$ & $\begin{array}{l}\text { Establece } \\
\text { diferencias entre } \\
\text { la señal ideal y } \\
\text { Real }\end{array}$ \\
\hline D & $\begin{array}{l}\text { Representa la } \\
\text { función utilizando } \\
\text { el Kmapa }\end{array}$ & $\begin{array}{l}\text { Dibuja el circuito } \\
\text { combinacional } \\
\text { utilizando los } \\
\text { operadores } \\
\text { lógicos básicos }\end{array}$ & $\begin{array}{l}\text { No realiza } \\
\text { los dibujos } \\
\text { esquemáticos } \\
\text { de los circuitos } \\
\text { combinacionales }\end{array}$ & $\begin{array}{l}\text { No contesta o } \\
\text { responde no } \\
\text { lo sé. }\end{array}$ \\
\hline $\mathbf{E}$ & $\begin{array}{l}\text { No contesta o } \\
\text { responde no lo sé. }\end{array}$ & $\begin{array}{l}\text { No contesta o } \\
\text { responde no } \\
\text { lo sé. }\end{array}$ & & \\
\hline
\end{tabular}

Una vez establecidos los modelos, se asignó a cada estudiante en base a las respuestas, la letra asociada al modelo de conocimiento para cada una de las preguntas tanto para el cuestionario inicial como para el final. De esta forma es posible observar la evolución del aprendizaje en todos los alumnos. El resultado de las 4 preguntas para los 9 estudiantes se muestra en la Tabla 5, donde es posible cuantificar la progresión de cada estudiante, observando así cómo ha evolucionado. En general se aprecia un progreso importante en el nivel de conocimientos de los estudiantes; todos han mejorado en diferentes porcentajes, hay alumnos cuya mejora es relativamente baja

Ciclos de Mejora en el Aula (2020). Experiencias de Innovación Docente de la US Esta obra se distribuye con la licencia Creative Commons 
debido a que ya se encontraban en el modelo establecido como más alto. Este caso me hace plantearme para futuros CIMAs alguna estrategia que le permita a estos estudiantes también evolucionar en su aprendizaje

Tabla 5. Progresión del aprendizaje de los estudiantes

\begin{tabular}{|c|c|c|c|c|c|c|c|c|c|c|}
\hline \multirow[t]{2}{*}{ Estudiante } & \multicolumn{8}{|c|}{ Preguntas } & \multirow[t]{2}{*}{ Puntuación } & \multirow[t]{2}{*}{ Mejora } \\
\hline & 11 & $1 F$ & 21 & $2 F$ & 31 & $3 F$ & 41 & $4 \mathrm{~F}$ & & \\
\hline \multirow[t]{2}{*}{1} & $\mathrm{D}$ & C & $\mathrm{E}$ & A & B & A & $\mathrm{D}$ & B & \multirow[t]{2}{*}{4.39} & \multirow[t]{2}{*}{$44 \%$} \\
\hline & \multicolumn{2}{|c|}{0.5} & \multicolumn{2}{|c|}{2.0} & \multicolumn{2}{|c|}{0.63} & \multicolumn{2}{|c|}{1.26} & & \\
\hline \multirow[t]{2}{*}{2} & $E$ & C & D & B & B & C & C & C & \multirow[t]{2}{*}{1.37} & \multirow[t]{2}{*}{$13.7 \%$} \\
\hline & \multicolumn{2}{|c|}{1.0} & \multicolumn{2}{|c|}{1.0} & \multicolumn{2}{|c|}{-.63} & \multicolumn{2}{|c|}{0.6} & & \\
\hline \multirow[t]{2}{*}{3} & D & C & $\mathrm{E}$ & B & A & A & $\mathrm{D}$ & B & \multirow[t]{2}{*}{3.26} & \multirow[t]{2}{*}{$32.6 \%$} \\
\hline & \multicolumn{2}{|c|}{0.5} & \multicolumn{2}{|c|}{1.5} & \multicolumn{2}{|c|}{0.0} & \multicolumn{2}{|c|}{1.26} & & \\
\hline \multirow[t]{2}{*}{4} & $C$ & B & $C$ & $\mathrm{~A}$ & $\mathrm{~B}$ & C & $\mathrm{B}$ & $\mathrm{A}$ & \multirow[t]{2}{*}{2.76} & \multirow[t]{2}{*}{$27.6 \%$} \\
\hline & \multicolumn{2}{|c|}{0.5} & \multicolumn{2}{|c|}{1.0} & \multicolumn{2}{|c|}{0.63} & \multicolumn{2}{|c|}{0.63} & & \\
\hline \multirow[t]{2}{*}{5} & $E$ & $\mathrm{~B}$ & $\mathrm{~B}$ & A & $\mathrm{B}$ & $\mathrm{B}$ & $\mathrm{D}$ & B & \multirow[t]{2}{*}{3.26} & $32.6 \%$ \\
\hline & & & & & & & & & & \\
\hline 6 & C & A & C & A & D & B & A & A & 3.26 & $32.6 \%$ \\
\hline & & & & & & & & & & \\
\hline 7 & B & A & $\mathrm{E}$ & C & C & $\mathrm{B}$ & $\mathrm{D}$ & C & 2.76 & $27.6 \%$ \\
\hline & & & & & & & & & & \\
\hline 8 & $E$ & B & B & A & $\mathrm{D}$ & $\mathrm{B}$ & $\mathrm{C}$ & A & 4.26 & $42.6 \%$ \\
\hline & & & & & & & & & & \\
\hline 9 & E & D & $\mathrm{E}$ & C & D & C & D & C & 2.63 & $26.3 \%$ \\
\hline & & & & & & & & & & \\
\hline
\end{tabular}

Los resultados también se han representado en forma de escaleras de aprendizaje para cada pregunta. Como ejemplo, se presenta la Figura 3 realizada para la pregunta 2 del cuestionario; en el rellano de la escalera aparece el modelo, así como el porcentaje de estudiantes en cada nivel tanto para el cuestionario inicial (verde) como para el final (azul). En la subida de cada escalón (izquierda) se encuentra el obstáculo que debe superar el estudiante para alcanzar el siguiente modelo o escalón. Los principales obstáculos para este caso, identificados gracias al análisis de sus respuestas, fueron cuatro. El primer obstáculo que Ciclos de Mejora en el Aula (2020). Experiencias de Innovación Docente de la US 
debe vencer el estudiante se encuentra entre el modelo $\mathrm{E}$ y $\mathrm{D}$, el cual está relacionado con el desconocimiento de la existencia de los operadores Lógicos Básicos. El segundo obstáculo se encuentra entre el modelo D y $C$ y es que el alumno no es consciente del operador NAND. Después, entre el modelo C y $\mathrm{B}$, el obstáculo está relacionado con la ignorancia del funcionamiento del operador NAND. Finalmente, el obstáculo que se encuentra entre el nivel B y el A depende de que el alumno razone las debilidades y fortalezas entre la NAND y los operadores lógicos básicos. Al analizar el progreso del conocimiento en los alumnos de esta manera, ha cambiado mi concepto de evaluar, lo que me lleva a tener en cuenta al momento de calificar la superación de obstáculos por parte de los alumnos. De igual forma, el identificar los principales obstáculos con los que se encuentran los estudiantes para obtener los conocimientos, ha sido un gran descubrimiento, ya que al determinar cuáles son, me ayuda a fomentar actividades específicas que contribuyan favorablemente a superarlos.

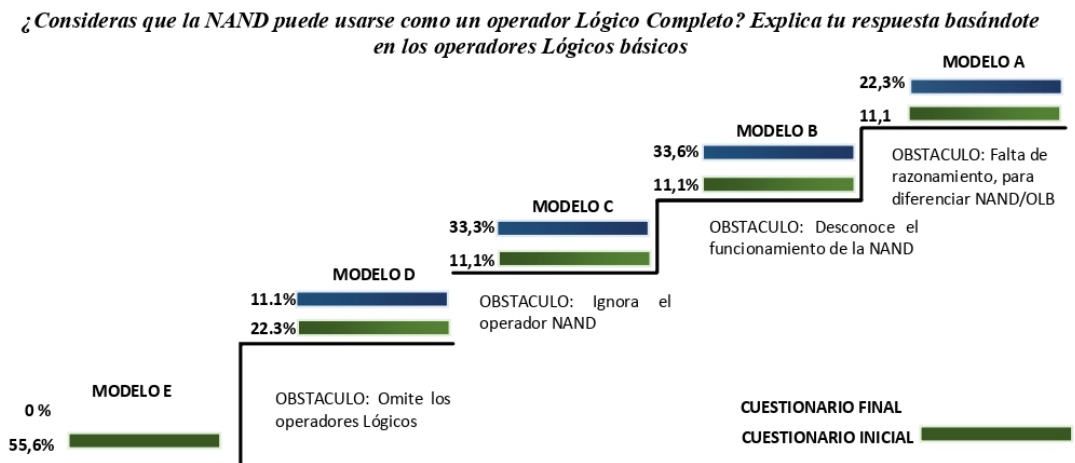

Figura 3. Escalera de aprendizaje para la pregunta 2

\section{Evaluación del Ciclo de Mejora Aplicado}

Las nuevas estrategias de actuación ensayadas en las prácticas de laboratorio me han mostrado que son más adecuadas que las que se aplicaban tradicionalmente. El Ciclos de Mejora en el Aula (2020). Experiencias de Innovación Docente de la US
Esta obra se distribuye con la licencia Creative Commons 
interés de los alumnos por buscar la verdad en el funcionamiento de los circuitos ha incrementado en un gran porcentaje, logrando mayor satisfacción en la práctica docente y un mejor ambiente en el laboratorio.

El trabajo que he realizado a lo largo del CIMA me ha hecho consiente de varias cosas: en primer lugar, de que en cualquier clase estoy aplicando una determinada metodología (Porlán, 2017), la cual la elijo yo. En segundo lugar, que los contenidos que enseñamos no solo se refieren a alguna disciplina, sino que se pueden distinguir tres grandes grupos: conceptuales, procedimentales y actitudinales (Porlán, 2017). Tercero; que aprender es un proceso de cambio relevante en los modelos y formas de razonar de las personas, por lo que para evaluar el aprendizaje es necesario acceder a los modelos mentales de los estudiantes (Porlán, 2017). La puesta en práctica de este ciclo de mejora me ha proporcionado algunas herramientas y, sobre todo, la motivación para iniciar un cambio progresivo en mi docencia que permita una mayor implicación del alumnado en su proceso de aprendizaje, de manera que aprendan más y mejor.

Me he dado cuenta de que no quiero seguir dando mis clases siguiendo el modelo tradicional transmisivo y voy a tener en cuenta de ahora en adelante lo siguiente:

- Comenzar la clase creando un entorno de aprendizaje crítico y natural; con la intención de que los alumnos despierten su curiosidad, específicamente a través de una pregunta intrigante (Bain, 2007).

- Incorporar el trabajo en grupo en la mayoría de las clases, ya que a través de los dos CIMAs da muy buenos resultados, motivando a los estudiantes y estimulándolos intelectualmente.

- Utilizar secuencias de actividades variadas, que no sigan siempre el mismo modelo (cambiando el tipo de actividades o el orden), para evitar que los alumnos se

Ciclos de Mejora en el Aula (2020). Experiencias de Innovación Docente de la US Esta obra se distribuye con la licencia Creative Commons 
acomoden demasiado y mantener un cierto nivel de tensión intelectual.

- Pedir siempre a los alumnos compromiso con su proceso de aprendizaje y con el desarrollo de la clase (Bain, 2007). Vincular ese compromiso, al menos al principio, con la calificación de alguna forma.

- En todas las clases plantear a los estudiantes alguna actividad intelectual que vaya más allá de escuchar y anotar (Finkel, 2008), que tengan que producir algún resultado, preferentemente escrito., de manera que puedan concretar sus ideas, cuestionárselas y razonarlas.

- Terminar cada tema con una pregunta que puedan considerar interesante, y no necesariamente contestarla (Bain, 2007). Darles la oportunidad de probar lo que han aprendido dejándoles una cuestión en la que aplicarlo, si lo desea.

\section{Principios Didácticos en los que se basa el Ciclo de Mejora de Aula}

Los Principios Didácticos que han fundamentado esta experiencia se basan principalmente en actividades en las que el alumno es el sujeto protagonista de su aprendizaje y el docente un orientador, las cuales deben tener en cuenta lo siguiente.

- Iniciar con el diseño de una metodología de enseñanza donde abunden actividades en las que los estudiantes desarrollen el proceso de investigación, es decir, formulen hipótesis, realicen la práctica de esas hipótesis, para llegar a sus propias conclusiones.

- Seguir con la definición de los contenidos que se desea que el alumno aprenda, con la premisa "menos es más", donde escogiendo menos contenidos se logra un mayor aprendizaje.

Ciclos de Mejora en el Aula (2020). Experiencias de Innovación Docente de la US Esta obra se distribuye con la licencia Creative Commons 
- Crear ambientes de aprendizaje interesantes y más reales para el alumno que logren efectos de enganche para obtener los mejores resultados.

- Explorar de forma anónima los modelos mentales de los estudiantes, a través de cuestionario iniciales y finales; para poder observar la evolución del aprendizaje y así determinar los obstáculos que ayuden a ajustar y mejorar el diseño.

- Incorporar diversos elementos y actividades de contraste que le permitan al docente guiar al alumno en la búsqueda de la verdad.

- Integrar instrumentos y herramientas para evaluar y tener una visión holística del proceso (diarios docentes, cuestionarios, tareas de clases, evidencias, herramientas tecnológicas, fotografías, vídeos, reflexiones de los estudiantes...).

Ciclos de Mejora en el Aula (2020). Experiencias de Innovación Docente de la US Esta obra se distribuye con la licencia Creative Commons 
Palabras clave: Circuitos electrónicos digitales, grado en Ingeniería Informática, docencia universitaria, experimentación docente universitaria, prácticas de laboratorio.

Key words: Digital Electronic Circuits (DEC), Computer Engineering Degree, University Teaching, Experimental University Teaching, Laboratory Practice.

\section{Bibliografía}

Bain, K. (2007). Lo que hacen los mejores profesores universitarios. Valencia: Publicaciones Universidad de Valencia.

Finkel, D. (2008). Dar clases con la boca cerrada. Valencia: Publicaciones Universidad de Valencia.

Porlán, R. (2017). Enseñanza Universitaria, Cómo Mejorarla. Madrid. Ediciones Morata.

Ciclos de Mejora en el Aula (2020). Experiencias de Innovación Docente de la US Esta obra se distribuye con la licencia Creative Commons 